\title{
Author Correction: Battery-free, wireless soft sensors for continuous multi-site measurements of pressure and temperature from patients at risk for pressure injuries
}

Yong Suk Oh (1), Jae-Hwan Kim, Zhaoqian Xie (1), Seokjoo Cho (1), Hyeonseok Han, Sung Woo Jeon, Minsu Park, Myeong Namkoong, Raudel Avila, Zhen Song, Sung-Uk Lee, Kabseok Ko, Jungyup Lee, Je-Sang Lee, Weon Gi Min, Byeong-Ju Lee (10, Myungwoo Choi, Ha Uk Chung, Jongwon Kim, Mengdi Han, Jahyun Koo, Yeon Sik Choi(D), Sung Soo Kwak, Sung Bong Kim, Jeonghyun Kim(D), Jungil Choi (D), Chang-Mo Kang,

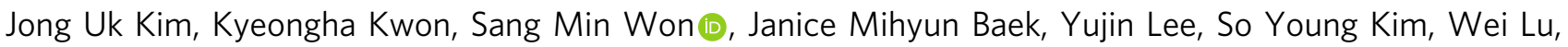
Abraham Vazquez-Guardado (1), Hyoyoung Jeong (1), Hanjun Ryu (1), Geumbee Lee, Kyuyoung Kim, Seunghwan Kim, Min Seong Kim, Jungrak Choi, Dong Yun Choi, Quansan Yang (1), Hangbo Zhao (iD, Wubin Bai (D), Hokyung Jang (1), Yongjoon Yu, Jaeman Lim (D), Xu Guo, Bong Hoon Kim, Seokwoo Jeon (1), Charles Davies (1D, Anthony Banks, Hyung Jin Sung, Yonggang Huang (1), Inkyu Park \& John A. Rogers (D)

Correction to: Nature Communications https://doi.org/10.1038/s41467-021-25324-w, published online 24 August 2021.

In the Acknowledgements section of this article the grant number relating to Korea Government (MSIT) given for Yong Suk Oh, Seokjoo Cho, Hyeonseok Han, Kyuyoung Kim, Seunghwan Kim, Min Seong Kim, Jungrak Choi, and Inkyu Park was incorrectly given as 2018R1A2B200491013 and should have been 2021R1A2C3008742. The original article has been corrected.

Published online: 18 November 2021

Open Access This article is licensed under a Creative Commons Attribution 4.0 International License, which permits use, sharing, adaptation, distribution and reproduction in any medium or format, as long as you give appropriate credit to the original author(s) and the source, provide a link to the Creative Commons license, and indicate if changes were made. The images or other third party material in this article are included in the article's Creative Commons license, unless indicated otherwise in a credit line to the material. If material is not included in the article's Creative Commons license and your intended use is not permitted by statutory regulation or exceeds the permitted use, you will need to obtain permission directly from the copyright holder. To view a copy of this license, visit http://creativecommons.org/licenses/by/4.0/.
}

(c) The Author(s) 2021 